\title{
Abordagem diagnóstica e terapêutica da sífilis gestacional e congênita: revisão narrativa
}

\author{
Diagnostic and therapeutic approach to gestational and congenital syphilis: narrative \\ review
}

Enfoque diagnóstico y terapéutico de la sífilis gestacional y congénita: revisión narrativa

Crislene de Oliveira Campos ${ }^{1 *}$, Crislane Oliveira Campos ${ }^{1}$.

\section{RESUMO}

Objetivo: Descrever as manifestações clínica, etiologia e transmissão da sífilis gestacional e congênita, bem como os critérios para o diagnóstico e recomendações terapêuticas. Revisão bibliográfica: A sífilis quando adquirida durante a gestação pode levar ao abortamento espontâneo, morte fetal ou neonatal, prematuridade e graves danos à saúde do concepto, como o comprometimento oftalmológico, auditivo e neurológico. O risco de transmissão vertical depende do estágio da infecção materna e da idade gestacional em que ocorre a exposição fetal, sendo de 70 a $100 \%$ a taxa de transmissão vertical observada em gestantes com sífilis recente e de 30 a $40 \%$ nos casos de sífilis tardia. O diagnóstico da sífilis, na ausência de manifestações clínicas, é feito por exames sorológicos. Para confirmar a quantidade de gestantes que apresentem o exame sorológico específico para a sífilis positivo, existem o VDRL, bem como o FTA/ ABS e teste rápido. Quando adquirida durante a gestação, a sífilis pode levar ao abortamento espontâneo, morte fetal ou neonatal, prematuridade e graves danos à saúde do concepto, como o comprometimento oftalmológico, auditivo e neurológico. Considerações finais: Percebe-se que a captação precoce e a adesão da gestante ao prénatal, assim como uma assistência de qualidade, dão oportunidade à gestante de receber informações e orientações que the permitam prevenir uma gravidez não planejada e proteger-se de infecções sexualmente transmissíveis.

Palavras-chave: Sífilis, Assistência pré-natal, Sífilis congênita.

\begin{abstract}
Objective: To describe the clinical manifestations, etiology, and transmission of gestational and congenital syphilis, as well as the criteria for diagnosis and therapeutic recommendations. Bibliographic review: Syphilis when acquired during pregnancy can lead to spontaneous abortion, fetal or neonatal death, prematurity, and serious damage to the health of the fetus, such as ophthalmological, auditory, and neurological impairment. The risk of vertical transmission depends on the stage of maternal infection and the gestational age at which fetal exposure occurs, with 70 to $100 \%$ being the vertical transmission rate observed in pregnant women with recent syphilis and 30 to $40 \%$ in cases of late syphilis. The diagnosis of syphilis, in the absence of clinical manifestations, is made by serological tests. To confirm the number of pregnant women who present the specific serological test for positive syphilis, there is the VDRL, as well as the FTA / ABS and rapid test. When acquired during pregnancy, syphilis can lead to spontaneous abortion, fetal or neonatal death, prematurity, and serious damage to the health of the fetus, such as ophthalmological, auditory and neurological impairment. Final considerations: It is noticed that the early capture and adherence of the pregnant woman to prenatal care, as well as quality care, give the pregnant woman the opportunity to receive information and guidance that will enable her to prevent an unplanned pregnancy and protect herself from infections sexually transmitted.
\end{abstract}

Key words: Syphilis, Prenatal care, Congenital syphilis.

1Universidade Ceuma (UNICEUMA), Imperatriz - MA. *E-mail: crislene_oc@hotmail.com

SUBMETIDO EM: 5/2020

ACEITO EM: 6/2020

PUBLICADO EM: 8/2020 


\section{RESUMEN}

Objetivo: Describir las manifestaciones clínicas, la etiología y la transmisión de la sífilis gestacional y congénita, así como los criterios diagnósticos y las recomendaciones terapéuticas. Revisión bibliográfica: La sífilis cuando se adquiere durante el embarazo puede conducir a un aborto espontáneo, muerte fetal o neonatal, prematuridad y daños graves a la salud del feto, como deterioro oftalmológico, auditivo y neurológico. El riesgo de transmisión vertical depende de la etapa de la infección materna y la edad gestacional en la que ocurre la exposición fetal, siendo el 70 al 100\% la tasa de transmisión vertical observada en mujeres embarazadas con sífilis reciente y del 30 al $40 \%$ en casos de sífilis tardía. El diagnóstico de sífilis, en ausencia de manifestaciones clínicas, se realiza mediante pruebas serológicas. Para confirmar el número de mujeres embarazadas que presentan la prueba serológica específica para sífilis positiva, existe el VDRL, además del TLC / ABS y la prueba rápida. Cuando se adquiere durante el embarazo, la sífilis puede conducir a un aborto espontáneo, muerte fetal o neonatal, prematuridad y daños graves a la salud del feto, como deterioro oftálmico, auditivo y neurológico. Consideraciones finales: Se observa que la captura y la adhesión temprana de la mujer embarazada a la atención prenatal, así como a la atención de calidad, le dan a la mujer embarazada la oportunidad de recibir información y orientación que le permitirán evitar un embarazo no planificado y protegerse de las infecciones transmitido sexualmente.

Palabras clave: Sífilis, Atención prenatal, Sífilis congénita.

\section{INTRODUÇÃO}

As infecções sexualmente transmissíveis (IST) se encontram entre as principais causas de doenças no mundo, constituindo-se uma grande preocupação, devido ao número de pessoas infectadas a cada ano. Estima-se aproximadamente 12 milhões de novos casos de pessoas infectadas por ano com alguma doença relacionada ao sexo, dentre as quais a sífilis tem grande representatividade.

A sífilis em gestante possibilita um risco maior de transmissão vertical para o feto. Alguns fatores podem contribuir para essa transmissão, por exemplo, a condição socioeconômica baixa das mulheres gestantes é um dos fatores determinantes para contrair e transmitir à sífilis. Além disso, observa-se que a baixa escolaridade pode contribuir a não adesão do tratamento. No entanto, mulheres acompanhadas no pré-natal têm menor risco de transmissão ao feto (FILGUEIRÓ-FILHO EA, et al., 2012).

No Brasil, a sífilis, ainda é um grande problema de saúde pública, apesar de ser uma doença de fácil diagnóstico e tratamento altamente eficaz. Dessa forma, mostra-se importante que os profissionais da saúde tenham acesso às informações demonstrativas da magnitude do problema, para que disponham de subsídios para planejar novas intervenções e estratégias voltadas a diminuir os índices da enfermidade (LINS CDM, 2014).

A sífilis é uma infecção causada pela bactéria Treponema pallidum; uma espiroqueta de transmissão sexual ou vertical que pode causar respectivamente a forma adquirida ou congênita da doença. É considerado um grave problema de saúde pública por sua alta prevalência e quando presente no período gestacional pode ocasionar muitos efeitos nocivos e transmissão vertical que resulta em sífilis congênita sendo responsável por altas taxas de mortalidade, além de acarretar graves consequências para o concepto (SOARES BGM, et al., 2017).

O Ministério da Saúde (2010) enfatiza que a maioria das pessoas com sífilis tende a não ter conhecimento da infecção, podendo transmitir aos seus parceiros sexuais. Isso ocorre devido à ausência de sintomatologia. Em contrapartida, quando não tratada, pode evoluir para formas graves e comprometer os sistemas nervoso e cardiovascular.

As manifestações da doença são divididas em três formas clínicas: primária, secundária e terciária. A fase primária é caracteriza-se pela presença do cancro duro, localizado em órgãos genitais, aparecendo entre 10 a 20 dias após o contato. A secundária caracteriza-se por linfadenopatias generalizadas, erupções cutâneas, úlceras rasas em mucosas da boca e órgãos sexuais e geralmente surge de duas a 10 semanas após 0 
aparecimento do cancro duro. A terciária aparece de 8 a 25 anos após a infecção inicial e caracteriza-se por apresentar sintomas neurológicos e cardiovasculares (BROSO MM, et al., 2013).

O diagnóstico laboratorial da sífilis se faz por técnicas variadas e depende da fase da infecção. Nas lesões de fase primária e algumas lesões da fase secundária pode ser realizada a pesquisa direta do Treponema pallidum. O exame microbiológico pode ser realizado também na placenta, cordão umbilical e nas lesões cutâneo-mucosas da criança.

Os testes sorológicos dividem-se em treponêmicos e não treponêmicos. Os testes treponêmicos confirmam a infecção com a detecção da presença de anticorpos anti-Treponema. Entre eles estão o FTAAbs (Fluorescent Treponema Antibody Absorvent Test), o MH-TP (Micro-Hemaglutinação para Treponema pallidum ou TPHA), o Elisa (teste imunoenzimático) e os testes imunocromatográficos (testes rápidos). Os testes não treponêmicos são importantes para o diagnóstico e o seguimento pós-terapêutico, os mais utilizados são o VDRL (Veneral Disease Research Laboratory) e o RPR (Rapid Plasm Reagin) (BRASIL, 2013).

A sífilis gestacional é uma doença de fácil diagnóstico e seu tratamento pode ser realizado com baixo custo e pouca ou nenhuma dificuldade operacional. Logo, requer intervenção imediata, para que se reduza ao máximo a possibilidade de transmissão vertical. Dessa maneira, é necessário no mínimo duas vezes na gestação a realização de testes, seja para parto ou curetagem uterina pós-abortamento (BRASIL, 2013). A sífilis afeta um milhão de gestantes por ano em todo o mundo, levando a mais de 300 mil mortes fetais e neonatais e colocando em risco de morte prematura mais de 200 mil crianças (BRASIL, 2017).

Os fetos de gestantes com sífilis não tratada podem apresentar manifestações clínicas precoce e tardia. A sífilis congênita precoce surge até o segundo ano de vida e podem evoluir com hepatomegalia, lesões cutâneas sofrimento respiratório, icterícia, anemia. Já a sífilis congênita tardia surge após o segundo ano de vida apresentando nariz "em sela", dentes incisivos medianos superiores deformados, mandíbula curta, arco palatino elevado, surdez neurológica e dificuldade no aprendizado (BRASIL, 2015).

O risco de transmissão vertical depende do estágio da infecção materna e da idade gestacional em que ocorre a exposição fetal, sendo de 70 a $100 \%$ a taxa de transmissão vertical observada em gestantes com sífilis recente e de 30 a $40 \%$ nos casos de sífilis tardia. A infecção é assintomática em mais de $50 \%$ dos recém-nascidos de mulheres com sífilis adquirida durante a gravidez, apresentando o surgimento dos primeiros sintomas aproximadamente com 3 meses de vida, o que intensifica a importância da triagem sorológica da gestante na maternidade (BARBOSA DRM, et al., 2017).

Diante do conhecimento do número elevado de sífilis gestacional e congênita, verificou-se a necessidade de maiores informações sobre a etiologia, transmissão, manifestações clínicas da doença bem como os critérios para o diagnóstico e recomendações terapêuticas. Assim, espera-se que esse estudo contribua para o conhecimento e entendimento da doença, permitindo um melhor planejamento das medidas de intervenção e prevenção nos grupos mais vulneráveis, e servir como ferramenta para auxiliar na avaliação das ações para a redução da transmissão vertical da sífilis.

\section{REVISÃO BIBLIOGRÁFICA}

\section{História da sífilis}

O termo sífilis originou-se de um poema, escrito pelo médico e poeta Girolamo Fracastoro em 1530, com o livro intitulado de Syphilis Sive Morbus Gallicus ("A sifilis ou mal gálico"). O livro narra à história de Syphilus, que foi punido com a doença sífilis por ter amaldiçoado o deus Apolo. Em 1546 Fracastoro levantou a hipótese que a sífilis fosse transmitida por via sexual, mas sua ideia não foi levada em consideração (BRASIL, 2010).

A sífilis é uma enfermidade conhecida desde o século XV, considerada como uma das principais pragas mundiais. Algumas hipóteses foram elaboradas para explicar sua origem. De acordo com a "hipótese colombiana", a sífilis teria sido introduzida na Europa pelos marinheiros espanhóis que participaram da descoberta da América. Outra hipótese seria que a sífilis seria originada de mutações sofridas por treponemas endêmicos do continente africano (AVELLEIRA JCR e BOTTINO G, 2006). 
Em 1905, o zoologista Fritz Schaudin e o dermatologista Paul Erich Hoffman descobriram o agente etiológico da sífilis, o Treponema pallidum. Os dois analisaram uma amostra de pápula existente na vulva de uma mulher com sífilis secundaria, ao microscópio observaram que os microrganismos que se moviam para frente e para trás e eram espiralados e finos. Primeiro denominara-os de Spirochaeta pallida e, um ano depois, denominou-se para Treponema pallidum. No Brasil, o surgimento da sífilis está associado à época do colonialismo, com a presença dos europeus, navios negreiros e índios (BRASIL, 2010; LINS CDM, 2014).

\section{Etiologia}

A Sífilis é uma doença infecciosa, causada pelo Treponema pallidum uma bactéria Gram-negativa do grupo das espiroquetas. O T. pallidum não possui membrana celular, é protegido por um envelope externo com três camadas ricas em moléculas de ácido $\mathrm{N}$-acetil murâmico e $\mathrm{N}$-acetil glucosamina. Sua estrutura é

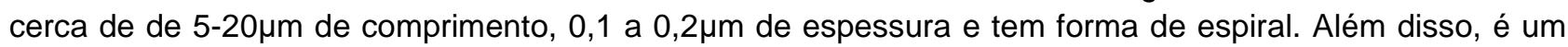
patógeno exclusivo do ser humano, apesar de, quando inoculado, causar infecções experimentais em macacos e ratos (AVELLEIRA JCR e BOTTINO G, 2006).

\section{Transmissão}

A transmissão é predominantemente pela via sexual e vertical pela placenta da mãe para o feto. A presença de processo inflamatório do colo uterino facilita à penetração dos Treponemas, que penetram a mucosa através de pequenas erosões ocorridas durante a relação sexual. Ademais, pode também ser transmitida por acidentes com pérfuro-cortantes e tatuagens. A transmissão da doença para o concepto pode ocorrer em qualquer trimestre da gestação, sendo mais provável que ocorra no segundo e terceiro trimestre. Além disso, durante a passagem do feto pelo canal vaginal existe a probabilidade de contaminação direta com T. pallidum, principalmente quando a gestante apresenta lesões genitais (LINS CDM, 2014; SONDA EC, et al., 2013).

\section{Formas da sífilis}

Considerando as manifestações clínica, temos: Sífilis primaria: Caracteriza-se por cancro duro, exulceração ou ulceração não dolorosa e de bordos elevados. É altamente infectante e rica em treponemas, que podem ser visualizados por meio de pesquisa direta em campo escuro. Após a infecção, ocorre um período de incubação entre 10 e 90 dias. Nas mulheres, geralmente a apresentação do cancro duro é comum no colo uterino ou na mucosa vaginal ou vulvar. No homem é mais comum no prepúcio, meato uretral ou mais raramente intra-uretral (LINS CDM, 2014; BRASIL, 2010; SALOMÃO R, 2017).

Sífilis secundária: Caracteriza- se pela disseminação da espiroqueta, quando não tratada. As manifestações clínicas geralmente são lesões em alvo plantares e palmares, alopecia irregular e/ou placas mucosas. Pode estar associada à febre, mal-estar, anorexia, cefaleia, mialgias e artralgias. As manifestações clínicas podem surgir de 4 a 10 semanas após o cancro duro aparecer (CUNNINGHAM FG, et al., 2012).

Sífilis latente: Caracteriza-se por desaparecimento dos sinais e sintomas após a sífilis secundária. Esse estágio pode ser classificado, de acordo com o tempo de infecção, em sífilis latente recente no primeiro ano e sífilis tardia quando permanece por mais de um ano de infecção. É importante diferenciar a fase latente da fase primária, para isso deve-se pesquisar no líquor a presença de anticorpos, utilizando-se o VDRL. Quando o VDRL é reagente no líquor acompanhado de baixos títulos no solo evidencia-se sífilis latente (BRASIL, 2010; SALOMÃO R, 2017).

Sífilis Terciária: Caracteriza-se por apresentar sintomas neurológicos (demência), cardiovasculares (aneurisma de aorta) e cutâneos (gomas). Geralmente ocorre de 3 a 12 anos após a fase de latência, manifestando-se na forma de inflamação e destruição de tecidos e ossos. Além disso, é caracterizada por tumorações amolecidas vistas na pele e nas membranas mucosas, que também podem acometer qualquer parte do corpo, inclusive no esqueleto ósseo (BRASIL, 2010; SALOMÃO R. 2017).

Sífilis cardiovascular: inflamação da aorta é o acometimento mais comum, a qual pode manifestar-se após 10 a 30 anos da infecção primária. A artéria mais acometida é a aorta ascendente, mas a maioria dos casos são assintomáticos. O aneurisma, estenose de artéria coronariana e insuficiência da válvula aórtica são as principais complicações de inflamação da aorta (AVELLEIRA JCR e BOTTINO G, 2006). 
Neurossífilis: após 12 a 18 meses da infecção pelo treponema pode ocorrer invasão das meninges, o quadro de neurossífilis se estabelece quando a infecção persiste. A neurossífilis pode ser assintomática ou sintomática. A assintomática é caracterizada pela ausência de sinais e sintomas neurológicos, mas com anormalidades no líquido cefalorraquidiano. A neurossifilis sintomática se caracteriza pela presença de paralisia geral progressiva e encefalite difusa com sinais focais (AVELLEIRA JCR e BOTTINO G, 2006).

\section{Diagnóstico}

Na sífilis primária o diagnóstico pode ser realizado pela visualização direta do treponema em campo escuro ou imunofluorescência direta. O teste sorológico é usado em pacientes assintomáticas ou para fins de rastreamento. Os testes sorológicos podem ser negativos no estágio inicial da sífilis, por isso a microscopia em campo escuro é o mais adequado para o diagnóstico da sífilis recente. Testes treponêmicos e não treponêmicos são as provas sorológicas mais utilizadas no diagnóstico da sífilis (CUNNINGHAM FG, et al., 2012; DAMASCENO ABA, et al., 2014).

Os testes não treponêmicos são importantes para o diagnóstico e o seguimento pós-terapêutico, os mais utilizados são o VDRL (Veneral Disease Research Laboratory) e o RPR (Rapid Plasm). Os testes treponêmicos confirmam a infecção com a detecção da presença de anticorpos anti-Treponema. Entre eles estão o FTA-Abs (Fluorescent Treponema Antibody Absorvent Test), o MH-TP (Micro-Hemaglutinação para Treponema pallidum ou TPHA), o Elisa (teste imunoenzimático), o Western blotting (WB) e os testes imunocromatográficos (testes rápidos) (DAMASCENO ABA, et al., 2014).

O teste rápido imunocromatográfico pode auxiliar no diagnóstico da doença, devido a leitura imediata. $O$ teste detecta anticorpos IgG, IgM e IgA contra um antígeno do $T$. pallidum, com sangue total ou gota obtida da ponta do dedo. O exame é simples, e pode ser realizado a leitura do teste em até 20 minutos após sua realização. No entanto, o teste não pode ser utilizado como critério exclusivo para o diagnóstico de sífilis (AVELLEIRA JCR e BOTTINO G, 2006; DAMASCENO ABA, et al., 2014).

No Brasil, durante o pré-natal o VDRL é o exame mais utilizado para rastreamento da doença, pois apresenta alta especificidade e sensibilidade, podendo permanecer reagente mesmo após a cura da doença. Recomenda-se a coleta do exame na primeira consulta de pré-natal, idealmente no primeiro trimestre, e o segundo na trigésima semana. $O$ terceiro VDRL deve ser realizado no momento da admissão hospitalar, seja por qualquer intercorrência na gestação e/ou assistência ao parto (CUNNINGHAM FG, et al., 2012; ZUGAIB M, 2016).

\section{Tratamento}

O tratamento da sífilis depende do seu estágio clínico. O medicamento padrão utilizado é a Penicilina Benzatina, principalmente para o tratamento da gestante e da doença fetal e também na prevenção da transmissão vertical. O fármaco age interferindo na síntese do peptidoglicano, componente da parede celular do T. pallidum. O resultado é a destruição do agente etiológico, resultante da entrada de água no Treponema (CUNNINGHAM FG, et al., 2012; LINS CDM, 2014)

A posologia da penicilina depende do estágio da doença. Na sífilis primária, secundária e latente recente é recomendado o uso de penicilina $\mathrm{C}$ benzatina, 2.400.000 unidades internacionais (UI) por via intramuscular, em dose única. Na sífilis latente tardia e terciária é recomendao o uso de penicilina $\mathrm{G}$ benzatina na dose de 2.400.000 UI por via intramuscular uma vez por semana, por 3 semanas. E na neurossífilis é recomendado 0 uso penicilina $C$ cristalina de 3.000 .000 a $4.000 .000 \mathrm{UI}$, por via intravenosa, a cada 4 horas por 10 a 14 dias, seguido de penicilina $\mathrm{G}$ benzatina, $2.400 .000 \mathrm{UI}$ por via intramuscular, semanalmente, por 3 semanas (ZUGAIB M, 2016)

No primeiro trimestre da gravidez, a terapêutica com penicilina costuma evitar a infecção fetal. Após esta fase, trata o concepto também. Em gestantes, o esquema terapêutico deverá ser administrado conforme o estágio da sífilis nas mesmas doses do tratamento padrão. $O$ tratamento somente é considerado eficaz, tanto para a mulher quanto para o feto, se administrado 30 dias antes do parto. Ademais, é necessário o tratamento do parceiro sexual, e uso de preservativos nas relações sexuais (LINS CDM, 2014). 
A gestante com alergia à penicilina, deverá ser dessensibilizada com a penicilina $\mathrm{V}$ oral. Só após 35 minutos do processo de dessensibilização que deverá ser feita a administração parenteral da penicilina. Entretanto, drogas alternativas deverão ser utilizadas, caso ocorra reações alérgicas. O esteorato de eritromicina poderá ser utilizado na dose de $500 \mathrm{mg}$, de seis em seis horas por quinze dias na sífilis primária, secundária e durante trinta dias na sífilis latente tardia. É valido ressaltar que esse medicamento não levará a cura do feto, somente da gestante (AVELLEIRA JCR e BOTTINO G, 2006; DAMASCENO ABA, et al., 2014).

\section{Sífilis congênita}

A Sífilis Congênita é uma patologia causada pelo Treponema pallidum, o qual se propaga por via hematogênica, infectando o feto através da placenta, devido à gestante infectada estar isenta de tratamento ou incorretamente tratada. A transmissão é possível em qualquer estágio da doença, sendo mais provável no primeiro ou segundo estágio da doença. Além disso, existe ainda a possibilidade de contaminação direta do agente etiológico para o feto, durante a passagem pelo canal de parto, uma vez que existam lesões genitais na gestante (SONDA EC, et al., 2013)

Quando a sífilis é adquirida durante a gravidez, poderá haver infecção sintomática ou assintomática em recém-nascidos. A maioria das crianças infectadas é assintomática ao nascimento, com surgimento dos primeiros sintomas nos primeiros três meses de vida. As manifestações clínicas da sífilis congênita variam conforme a classificação da doença. Esta enfermidade apresenta dois estágios: precoce e tardia. A sífilis congênita precoce surge até o segundo ano de vida e deve ser diagnosticada através de uma avaliação epidemiológica da situação materna, avaliações clínica e laboratorial e estudos de imagem na criança (BRASIL, 2006).

As principais características dessa síndrome são: hepatomegalia com ou sem esplenomegalia, lesões cutâneas, pseudoparalisia dos membros, sofrimento respiratório com ou sem pneumonia, rinite serosanguinolenta, icterícia, anemia e linfadenopatia generalizada e outros. A sífilis congênita tardia surge após o segundo ano de vida. O diagnostico também deve ser estabelecido por meio da associação de critérios epidemiológicos, clínicos e laboratoriais. As principais características desse estágio são: surdez neurológica e dificuldade no aprendizado, nariz "em sela", dentes incisivos medianos superiores deformados (dentes de Hutchinson), rágades periorais, arco palatino elevado, mandíbula curta (BRASIL, 2006).

Quando adquirida durante a gestação, a sífilis pode levar ao abortamento espontâneo, morte fetal ou neonatal, prematuridade e graves danos à saúde do concepto, como o comprometimento oftalmológico, auditivo e neurológico. Ademais, a partir da análise dos artigos selecionados, verificou-se que dentre os principais fatores associados à ocorrência da sífilis congênita entre as gestantes foram à idade materna menor de 20 anos, baixa escolaridade, início tardio do pré-natal, ter realizado menos de seis consultas e a não realização do VDRL.

A assistência pré-natal é fundamental à saúde materno-infantil. Nesse período, devem ser desenvolvidas atividades relacionadas à promoção da saúde e identificação de riscos para a gestante e o concepto, permitindo assim a prevenção de inúmeras complicações, além de reduzir ou eliminar fatores e comportamentos de risco associados a vários agravos à saúde (NONATO SM, et al., 2015; NASCIMENTO MID, et al., 2012).

\section{CONSIDERAÇÕES FINAIS}

Percebe-se que a captação precoce e a adesão da gestante ao pré-natal, assim como uma assistência de qualidade, dão oportunidade à gestante de receber informações e orientações que lhe permitam prevenir uma gravidez não planejada e proteger-se de infecções sexualmente transmissíveis. Desigualdades sociais e regionais no acesso aos serviços de saúde, associada a outros defeitos na assistência, como baixa cobertura no tratamento do parceiro, colaboram para a persistência da sífilis congênita como relevante problema de saúde pública no Brasil. A penicilina continua a ser a droga de escolha para o tratamento, assegurando a cura. Ainda que o diagnóstico e o tratamento sejam de fácil acesso e de baixo custo, a sífilis congênita permanece sendo um desafio da saúde pública e deve continuar sendo meta de estudos que gerem novas estratégias de prevenção. 


\section{REFERÊNCIAS}

1. AVELLEIRA JCR, BOTTINO G. Sífilis: diagnóstico tratamento e controle. Anais Brasileiros de Dermatologia, Rio de Janeiro, 2006; 81(2):111-26.

2. BARBOSA DRM, et al., Perfil Epidemiológico dos Casos de Sífilis Gestacional. Revista enfermagem UFPE online. Recife, 2017; 11(5): 1867-1874.

3. BRASIL MS. Secretária de Atenção à Saúde. Departamento de Atenção Básica. Atenção ao pré-natal de baixo risco. Brasília: Editora do Ministério da Saúde, 2013; 318p.

4. BRASIL MS. Secretaria de Vigilância em Saúde. Departamento de Vigilância, Prevenção e Controle das IST, do HIV/Aids e das Hepatites Virais. Boletim Epidemiológico de Sífilis. v. 48, n. 36, 2017; 48(36): 4-41.

5. BRASIL MS. Secretaria de Vigilância em Saúde. Departamento de DST, Aids e Hepatites Virais. Protocolo Clínico e Diretrizes Terapêuticas para Atenção Integral às Pessoas com Infecções Sexualmente Transmissíveis. Brasília: Ministério da Saúde, 2015;120p.

6. BROSO MM, et al., Detecção de sífilis congênita em pacientes atendidos na rede particular de saúde em Jundiaí, SP. Journal of the Health Sciences Institute, São Paulo, 2013; 31(4): 355-359.

7. BRASIL MS. Sífilis: Estratégias para Diagnóstico no Brasil. Brasília: Ministério da Saúde, Coordenação de Doenças Sexualmente Transmissíveis e Aids. 2010; 100p.

8. BRASIL MS. Secretária de Vigilância em Saúde. Programa Nacional de DST/AIDS. Diretrizes para controle da sífilis congênita: manual de bolso. Brasília: Editora do Ministério da Saúde, 2006; 72 p.

9. CUNNINGHAM FG, et al., OBSTETRÍCIA DE WILLIAMS. 23. Ed. Porto Alegre: AMGH, 2012; 1385 p.

10. DAMASCENO ABA. et al., Sífilis na gravidez. Revista HUPE, Rio de Janeiro, 2014; 13(3): 88-94.

11. FIGUEIRÓ-FILHO EA, et al., Sífilis e gestação: estudo comparativo de dois períodos (2006 e 2011) em população de puérperas. J Bras Doenças Sex Transm., Rio de Janeiro, 2012; 2(1): 32-37.

12. LINS CDM. Epidemiologia da Sífilis Gestacional e Congênita no Extremo Setentrional da Amazônia. 2014. Dissertação de Conclusão de Mestrado (Pós- Graduação em Ciências da Saúde) - Universidade Federal de Roraima, Boa Vista, 2014; $21 p$.

13. NASCIMENTO MID, et al., Gestações complicadas por sífilis materna e óbito fetal. Rev Bras Ginecol Obstet. Rio de Janeiro, 2012; 34(2):56-62.

14. NONATO SM, et al., Sífilis na gestação e fatores associados à sífilis congênita em Belo Horizonte-MG, 2010-2013. Epidemiol. Serv. Saúde, Brasília, 2015; 24(4):681-694.

15. SALOMÃO R. Infectologia: Bases clínicas e tratamento. - 1. ed. - Rio de Janeiro: Guanabara Koogan, $2017 ; 1629$ p.

16. SOARES BGM, et al., Perfil das Notificações de Casos de Sífilis Gestacional e Sífilis Congênita. SANARE - Revista de Políticas Públicas. Sobral, 2017; 16(2): 51-59.

17. SONDA EC, et al. Sífilis Congênita: uma revisão da literatura. Rev Epidemiol Control Infect. Rio Grande do Sul, 2013; $3(1): 28-30$.

18. ZUGAIB M. Zugaib obstetrícia. 3. Ed. Barueri: Manole, 2016; 1329 p. 